\title{
Interfaces and nanostructures of functional oxide octahedral framework structures
}

\author{
Felip Sandiumenge ${ }^{1 *}$, Núria Bagués ${ }^{1,2}$ and José Santiso ${ }^{2}$ \\ 1 Institut de Ciència de Materials de Barcelona (ICMAB-CSIC), Barcelona, Spain \\ 2 ICN2 - Institut Català de Nanociència i Nanotecnologia (CSIC-ICN), Barcelona, Spain
}

\section{Edited by:}

P. Davide Cozzoli, University of

Salento, Italy

Reviewed by:

Binping Xie, Fudan University, China

Taro Hitosugi, Tohoku University,

Japan

*Correspondence:

Felip Sandiumenge, Institut de Ciència de Materials de Barcelona, (ICMAB-CSIC), Campus de la UAB, Bellaterra, Barcelona 08193, Spain

e-mail: felip@icmab.es
In the past decade, the rich physics exhibited by solid interfaces combining octahedral framework structures of transition-metal oxides has fascinated the materials science community. However, their behavior still eludes the current understanding of classical semiconductor and metal epitaxy. The reason for that is rooted in the surprising versatility of linked coordination units to adapt to a dissimilar substrate and the strong sensitivity of strongly correlated electron oxides to external perturbations. The confluence of atomic control in oxide thin film epitaxy, state of the art high spatial resolution characterization techniques, and electronic-structure computations, has allowed in recent years to obtain first insights on the microscopic mechanisms governing the epitaxy of these complex materials.

Keywords: framework structures, strongly correlated electron oxides, perovskite, epitaxy, self-organization, strain, polar discontinuity, functional properties

\section{INTRODUCTION}

Interfaces bridging complex metal oxides have emerged in recent years as a new paradigm of epitaxy owing to their extraordinary potential to exhibit unanticipated and conceptually challenging states such as highly conducting electron gases between two insulating materials (Ohtomo and Hwang, 2004), interfacial superconductivity (Reyren et al., 2007; Gozar et al., 2008), or polarization-dependent spin transfer (Garcia et al., 2010). This fascinating behavior stems from the delicate balance existing among strongly coupled lattice, charge, spin, and orbital degrees of freedom, which in turn manifests a strong sensitivity to external perturbations (Hwang et al., 2012), such as the biaxial stress induced by a mismatched substrate, polar discontinuities, and even very subtle effects like slight dissimilarities in the orientation of the coordination units. These compounds commonly exhibit an $\mathrm{ABO}_{3}$ perovskite-type crystal structure characterized by a framework of corner sharing $\mathrm{BO}_{6}$ oxygen octahedra hosting the $\mathrm{B}$ cation that can be derived from a common undistorted cubic aristotype through the tilting of essentially rigid $\mathrm{BO}_{6}$ units (Glazer, 1972; Howard and Stokes, 1998). As illustrated in Figure 1A, considering that the coordination units are rigid but free to rotate about their shared corners, one obtains an articulated framework that may exhibit auxetic behavior (Alderson and Evans, 2002). In this idealized case, the misfit strain directly couples with octahedral tilts, which in turn control the chemical bonding and the physical properties through changes in the overlap between the B-site $3 \mathrm{~d}$ and oxygen 2p orbitals (Tokura and Nagaosa, 2000). Thus, controlling the topology of the octahedral framework through misfit strain appears as an obvious strategy to control the stability of electronic and magnetic states. In practice, however, the deformation of the framework appears to be governed by the relative rigidity between the $\mathrm{B}-\mathrm{O}-\mathrm{B}$ angles bridging adjacent octahedra and the $\mathrm{B}-\mathrm{O}$ bonds. The latter, in turn, can be distorted either at the expense of elastic or electronic energies depending on the electronic structure of the transition metal. As a consequence, a deterministic manipulation of interfacial structure and misfit strain in these complex materials demands simultaneous control on electronic (spin, charge, orbital) and lattice (elastic, octahedral tilting) degrees of freedom. Here, we review these mechanisms and highlight their potential in the design of self-organized oxide nanostructures with enhanced functionalities.

\section{INTERFACIAL EFFECTS}

Interfaces combining octahedral frameworks of transition-metal oxides tend to react against film/substrate dissimilarities in a cooperative way. This is because the various degrees of freedom of the system are associated with similar energies and therefore there is not a unique leading mechanism. To illustrate a common scenario, Figure 1B exemplifies the types of dissimilarities found between the canonic room temperature half-metal ferromagnet $\mathrm{La}_{0.7} \mathrm{Sr}_{0.3} \mathrm{MnO}_{3}$ (Urushibara et al., 1995) and the commonly used (001) oriented $\mathrm{SrTiO}_{3}$ substrate. In the following subsections, we briefly analyze the consequences of the various dissimilarities on the topology and the electronic structure of the epitaxial framework.

\section{MISFIT STRAIN}

Rhombohedral perovskites like $\mathrm{La}_{0.7} \mathrm{Sr}_{0.3} \mathrm{MnO}_{3}$ (space group R$3 c)$ are characterized by equal antiphase tilts of the $\mathrm{BO}_{6}$ octahedra about the three Cartesian axes, $\alpha=\beta=\gamma$ (see Figure 1A), resulting in a net rotation $\omega$ about the pseudocubic [111] direction $\alpha$ $=\beta=\gamma=\omega / 3^{1 / 3}$ (Haun et al., 1989). According to Glazer, 1972, this octahedral tilt pattern is noted as $a^{-} a^{-} a^{-}$, where the components of the triad describe the octahedral tilts about the unit cell axes of the undistorted cubic aristotype (Pm-3m, $\left.a^{0} a^{0} a^{0}\right)$. As a result of this tilting, the unit cell interaxial angles, $\alpha_{c}$, are no longer $90^{\circ}$ (Megaw and Darlington, 1975), inducing a misfit shear strain $\chi=\tan \left(\alpha_{c}-90^{\circ}\right)$ relative to the underlying (001) plane of 
A

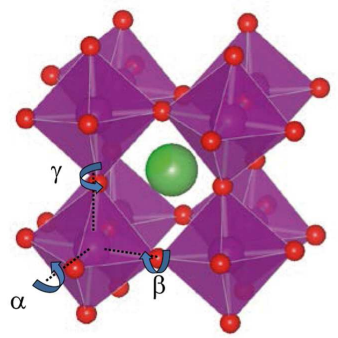

C

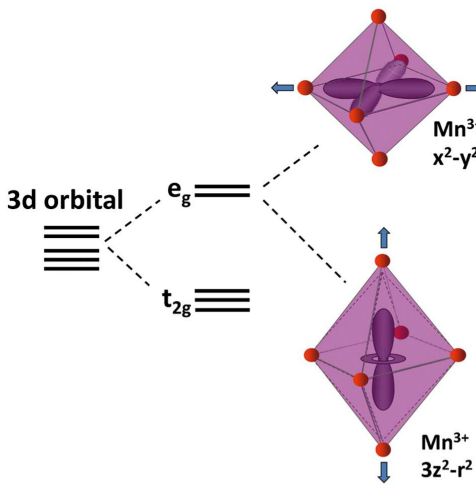

B Misfit strain Tilt system

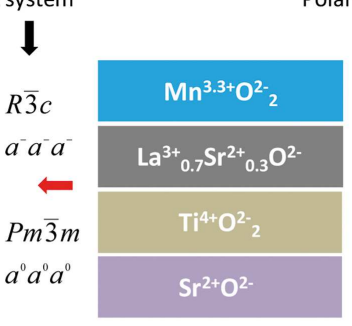

Polar discontinuity

\section{$\delta \sim 0.6 \%$}

$\chi \sim 0.7 \%$

\section{,}

,

.

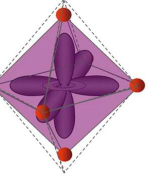

1 $\mathrm{Mn}^{3+} / \mathrm{Mn}^{4+}$

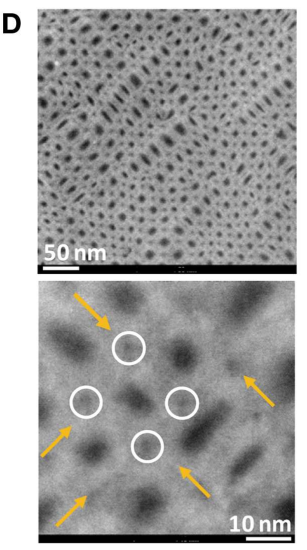

E
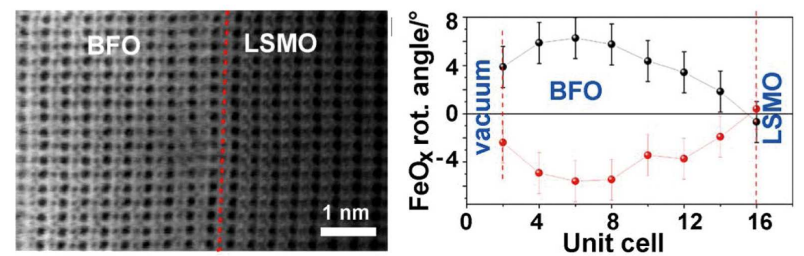

FIGURE 1 | (A) Pseudocubic (distorted) unit cell of an $\mathrm{ABO}_{3}$ perovskite. The aristotype structure (space group $P m-3 m$ ) consists of a three-dimensional framework of untilted $\left(\alpha=\beta=\gamma=0\right.$ ) regular $\mathrm{BO}_{6}$ octahedra ( $\mathrm{O}$ atoms are red circles) within the cavities of which lie the A cations (large green circles). Group-theoretical analysis yields 15 tilt systems (Howard and Stokes, 1998), which are commonly noted following the Glazer notation by the manner in which adjacent octahedra rotate one relative to each other along a particular axis of the undistorted cubic unit cell (Glazer, 1972); for instance, $a^{-(+)}$ indicates out-of-phase (in-phase) tilts and $a^{0}$ indicates no tilt. Thus the Pm-3m space group corresponds to the $a^{0} a^{0} a^{0}$ tilt system, while $\alpha \neq \beta \neq \gamma \neq 0^{\circ}\left(a^{-} b^{-} c^{-}\right)$ corresponds to the triclinic space group $P-1$. (B) Different sources of dissimilarity between $\mathrm{La}_{0.7} \mathrm{Sr}_{0.3} \mathrm{MnO}_{3}$ and $\mathrm{SrTiO}_{3}$. The rhombohedral structure of $\mathrm{La}_{0.7} \mathrm{Sr}_{0.3} \mathrm{MnO}_{3}$ is forced to match the cubic $\mathrm{SrTiO}_{3}$ substrate. The film is submitted to a tensile misfit strain besides a shear strain and a discontinuity between the $a^{-} a^{-} a^{-}$and $a^{0} a^{0} a^{0}$ tilt systems. Moreover, as indicated in the right column, the (002) planes in $\mathrm{La}_{0.7} \mathrm{Sr}_{0.3} \mathrm{MnO}_{3}$ have alternating net charges, while the (002) planes in $\mathrm{SrTiO}_{3}$ are neutral, resulting in a polar discontinuity at the interface. (C) The six $\mathrm{O}^{2-}$ anions surrounding the $\mathrm{Mn}^{3+/ 4+}$ cations in $\mathrm{La}_{0.7} \mathrm{Sr}_{0.3} \mathrm{MnO}_{3}$ create an octahedral crystal-field potential that partly lifts the degeneracy of the $3 \mathrm{~d}$ one-electron orbital levels. Orbitals pointing to the oxygen ions, $e_{g}, 3 z^{2}-r^{2}$ and $x^{2}-y^{2}$, are higher in energy than those pointing between them, $\mathrm{t}_{2 \mathrm{~g}}: x y, y z, z x$ (not shown). According to recent findings
(Pesquera et al., 2012), a tensile misfit strain would favor the preferential occupation of in-plane $x^{2}-y^{2}$ orbitals, while a compressive misfit strain would favor the occupation of the out-of-plane $3 z^{2}-r^{2}$ ones. Near the polar $\mathrm{La}_{0.7} \mathrm{Sr}_{0.3} \mathrm{MnO}_{3} / \mathrm{SrTiO}_{3}$ interface, the $\mathrm{Mn}^{3+} / \mathrm{Mn}^{4+}$ ratio is high and the strong localization of the $e_{g}$ electron gives rise to a measurable deformation of the $\mathrm{MnO}_{6}$ octahedra that contributes to the relaxation of the misfit strain. As the $\mathrm{Mn}^{3+} / \mathrm{Mn}^{4+}$ ratio is decreased away from the interface, the delocalized $e_{g}$ electron does not give rise to any measurable distortion of the coordination environment and the octahedra submitted to a misfit strain deform elastically (Sandiumenge et al., 2013). (D) Planar view annular dark field images of the misfit dislocation network in a $7.1 \mathrm{~nm}$ thick $\mathrm{La}_{0.7} \mathrm{Sr}_{0.3} \mathrm{MnO}_{3}$ film grown on a $\mathrm{LaAlO}_{3}$ substrate. The images have been obtained under low angle annular dark field (LAADF) conditions in order to emphasize the strain contrast. The images clearly reveal a self-organized strain pattern associated to the misfit dislocation network. The magnified bottom image shows in detail the correlation between the dislocations (yellow arrows indicate the positions of dislocations and circles mark their intersections) and the strain modulation.

(E) Bright field scanning transmission electron microscopy image of a $\mathrm{BiFeO}_{3} / \mathrm{La}_{0.7} \mathrm{Sr}_{0.3} \mathrm{MnO}_{3} / \mathrm{SrTiO}_{3}$ heterostructure, and evolution of octahedral tilt angles. The two curves correspond to the antiphase tilting of alternate octahedra. (E) Modified from Borisevich et al. (2010a), copyright 2010, American Physical Society. 
the cubic substrate. Note that since the substrate does not impose constrains on the inclination of the out-of-plane pseudocubic axis, the initial $-3 \mathrm{~m}$ rhombohedral point symmetry is reduced to a $2 / \mathrm{m}$ monoclinic one (Daumont et al., 2010). Therefore, the total strain energy of the rhombohedral film, $E_{\mathrm{T}}$, splits into two contributing elastic energies, a shear and a normal one, $E_{\mathrm{T}}=E(\chi)+E(\delta)$. In the absence of further perturbations at the interface, and assuming that the lattice distorts elastically (i.e., that strain couples with the B-O bonds, with no octahedral rotations), dislocation models (Matthews, 1975) predict a relaxation of $E(\delta)$ at critical thickness of $\sim 10 \mathrm{~nm}$ (Abellán et al., 2009), in strong contrast with the observation of fully strained in-plane lattice parameters up to $475 \mathrm{~nm}$ (Sandiumenge et al., 2013). Conversely, $E(\chi)$ is expected to be relieved from the initial growth stages by twinning on the (100) and (010) planes perpendicular to the interface (Farag et al., 2005), again in conflict with experimental observations pointing the occurrence of a shear transition at a critical thickness of about $2.5 \mathrm{~nm}$ (Sandiumenge et al., 2013; Santiso et al., 2013). Such discrepancies constitute in fact a dramatic manifestation of the ability of octahedral frameworks to propagate interfacial perturbations over length scales exceeding a few $100 \mathrm{~nm}$ at a lower energy cost than that associated with the formation of the misfit dislocation network needed to plastically relax an equivalent elastic strain energy.

Misfit strains have important consequences on the electronic structure of the films. As shown in Figure 1C, the octahedral coordination of the Mn-sites splits the $3 \mathrm{~d}$ orbitals into a degenerate $\mathrm{t}_{2 \mathrm{~g}}$ triplet and a degenerate $\mathrm{e}_{\mathrm{g}}$ doublet with $x y / y z / x z$ and $x^{2}-y^{2} / 3 z^{2}-r^{2}$ symmetries, respectively. Misfit strains induce further lowering of the symmetry that also unfolds the $e_{g}$ doublet. This is commonly achieved by half-filling of the $e_{g}$ orbitals, in which case the strong electron-phonon coupling induces a JahnTeller distortion of the coordination environment that breaks the degeneracy of the $x^{2}-y^{2}$ and $3 z^{2}-r^{2}$ orbitals, thus modifying their electron occupancy and eventually leading to orbital reconstructions. In the $\mathrm{La}_{1-\mathrm{x}} \mathrm{Sr}_{\mathrm{x}} \mathrm{MnO}_{3}$ solid solution, the electron occupancy of the $e_{g}$ doublet depends on the strength of the JahnTeller distortion, which in turn is determined by the hole doping level, $x$ (Tokura and Nagaosa, 2000). In epitaxial thin films, however, similarly to the Jahn-Teller distortion, misfit strains have the ability to break the $x^{2}-y^{2} / 3 z^{2}-r^{2}$ orbital degeneracy (Fang et al., 2000) as recently confirmed by X-ray linear dichroism investigations (Huijben et al., 2008; Tebano et al., 2008; Pesquera et al., 2012). A thorough investigation taking into account the attenuation of the signal with the depth in the sample, suggests that the observed X-ray linear dichroism is rather a consequence of the superposition of interfacial and free surface effects (Pesquera et al., 2012). As shown in Figure 1C, those results suggest that a tensile strain would favor the occupancy of the in-plane $x^{2}-y^{2}$ orbitals, while a compressive strain would induce the occupancy of the out-of-plane $3 z^{2}-r^{2}$ ones, as expected from electrostatic arguments. On the other hand, the $\mathrm{Mn}^{3+}$ cations located at the free surface appear to exhibit a preferred $3 z^{2}-r^{2}$ occupancy regardless of the sign of the interfacial biaxial strain. According to theoretical work (Calderon et al., 1999), this scenario is promoted by the absence of the apical oxygen in the coordination environment of surface cations and the resulting reduction of the repulsive electron-electron interaction between the manganese $3 \mathrm{~d}$ $\left(3 z^{2}-r^{2}\right)$ and oxygen $2 \mathrm{p}(z)$ orbitals.

Despite the surprising adaptability of the octahedral framework, there are situations where plastic relaxation is energetically favored. As an example, $\mathrm{La}_{0.7} \mathrm{Sr}_{0.3} \mathrm{MnO}_{3}$ films grown on $\mathrm{LaAlO}_{3}$ substrates under a compressive strain of $-2.3 \%$ are relaxed by misfit dislocations at a critical thickness of about $3 \mathrm{~nm}$. This indicates that there is a limited range of rigid octahedral tilts within which the film can deform to fit the substrate. In this case, the value of the out-of-plane lattice parameter prior to the onset of plastic relaxation, 3.98(1) $\AA$, is consistent with the value derived from the Poisson's ratio $\nu=0.33,3.975 \AA$, indicating that the film behaves elastically. Note that on a rigid octahedron basis, see Figure 1A, a shrinking of the in-plane lattice parameters can be either accomplished by increasing the octahedral tilt $\gamma$ about the $c$-axis, or by increasing the octahedral tilts simultaneously about the in-plane $a$ and $b$ axes. While the first operation would leave the length of the $c$-axis unaltered, the latter would even shrink it resulting in a strong contraction of the unit cell volume. Figure 1D shows the strain pattern associated with the misfit dislocation structure of a $7.1 \mathrm{~nm}$ thick film projected on the interface plane, using annular dark field scanning transmission electron microscopy at low beam convergence angles (Fitting et al., 2006). The density of the pattern can be manipulated through the thickness of the film. Thus, misfit dislocations in such compounds provide a means for the self-organization and spatial modulation of strain patterns in a strongly susceptible functional matrix, expanding the possibilities of interfacial engineering beyond homogeneous manipulations of the octahedral pattern. Moreover, dislocations in complex oxides are known to exhibit distinct electrical properties compared to the host material (Szot et al., 2006). Although the control of their density and distribution has been extensively studied in other functional oxides in bulk form (Sandiumenge et al., 2000), the spontaneous formation of regular patterns at interfaces in heteroepitaxial systems offers unique opportunities for miniaturization.

\section{POLAR DISCONTINUITIES}

Besides misfit strains, polar discontinuities are a common driving force for interfacial charge and orbital reconstructions. They appear as a result of the fact that individual atomic layers $\mathrm{AO}$ and $\mathrm{BO}_{2}$ in $\mathrm{ABO}_{3}$ perovskites can be electrically charged, as is the case of $\mathrm{La}_{0.7} \mathrm{Sr}_{0.3} \mathrm{MnO}_{3}$ (see Figure 1B). The net charge per area unit is $+2 / 3 \mathrm{e}$ and $-2 / 3 \mathrm{e}$ in $\mathrm{La}_{0.7} \mathrm{Sr}_{0.3} \mathrm{MnO}_{3}$, while it is null in $\mathrm{SrTiO}_{3}$ ( $\mathrm{e}$ is the electron charge). Polar discontinuities lead to a diverging electrostatic potential which in the absence of any reconstruction results in the so-called polar catastrophe (Nakagawa et al., 2006). Since $\mathrm{La}_{0.7} \mathrm{Sr}_{0.3} \mathrm{MnO}_{3}$ exhibits metallic behavior, mobile charges can screen the diverging potential (Dagotto, 2009), resulting in a compensating charge transfer of $1 / 3 \mathrm{e}$. This would lead to a shift of the Mn valency at the interface over a screening length of a few nanometers toward $3+$, as confirmed by electron energy loss (Riedl et al., 2009), X-ray absorption (Lee et al., 2010), and $\mathrm{x}$-ray photoemission (Sandiumenge et al., 2013) spectroscopies. According to the bulk $\mathrm{La}_{1-\mathrm{x}} \mathrm{Sr}_{\mathrm{x}} \mathrm{MnO}_{3}$ phase diagram (Dabrowski et al., 1999), the transfer of charge toward the interface results in the formation of a non-ferromagnetic and insulating wetting layer called "dead-layer." As films thicken beyond $\sim 2.5 \mathrm{~nm}$, the 
number of $e_{\mathrm{g}}$ electrons decreases and become delocalized with strong consequences on the deformation behavior of the films; the electronic energy gained by removing the $e_{g}$ orbital degeneracy becomes progressively exceeded by the elastic energy opposing a similar expansion of the equatorial $\mathrm{Mn}-\mathrm{O}$ distances needed to keep the in-plane lattice parameters fully strained (see Figure 1C). This unbalance evolves until a fully elastically strained rhombohedral phase condenses at a thickness of 10-20 nm, similar to the critical thickness for plastic relaxation. Strikingly, above this thickness the elastic energy is released by a composite octahedral tilting mechanism, which allows to maintain fully strained in-plane lattice parameters up to at least $475 \mathrm{~nm}$ (Sandiumenge et al., 2013). This complex behavior clearly reflects the competing nature of the different degrees of freedom involved in the relaxation mechanism of such materials.

Thanks to the extraordinary progress achieved in the atomic control of interfaces in oxide heterostructures, the formation of the dead-layer can be avoided by precise modifications of the interfacial architecture (Boschker et al., 2012), or even polar discontinuities can be exploited to intentionally induce hole doping avoiding disorder effects typically associated with chemical doping (Dagotto, 2009).

\section{OCTAHEDRAL TILT DISCONTINUITIES}

A more subtle dissimilarity is that between the octahedral tilt systems of the film and the substrate. The concept of interfacial octahedral coupling resides in the idea that the substrate tends to transmit its octahedral pattern to the film. This concept has motivated an intense research on its use for the engineering of novel tilt patterns with specific functionalities (Rondinelli and Spaldin, 2011; Rondinelli et al., 2012; Moon et al., 2014). Excitingly, recent advances in electron microscopy have provided direct access to oxygen positions (Jia et al., 2009; Borisevich et al., 2010a), and though direct experimental evidences are still very scarce, indeed they support the idea of octahedral coupling across the interfaces.

As an example, Figure 1E shows the variation of octahedral tilt angles in a $\mathrm{BiFeO}_{3}$ film grown on a $5 \mathrm{~nm} \mathrm{La} \mathrm{La}_{0.7} \mathrm{Sr}_{0.3} \mathrm{MnO}_{3}$ buffer layer fully elastically strained on top of $\mathrm{a} \mathrm{SrTiO}_{3}$ substrate, as determined by bright field scanning transmission electron microscopy imaging (Borisevich et al., 2010a). As can be seen in the right panel, the in-plane octahedral tilts of the $\mathrm{La}_{0.7} \mathrm{Sr}_{0.3} \mathrm{MnO}_{3}$ buffer are suppressed by the influence of the cubic $\mathrm{SrTiO}_{3}$ substrate, and this pattern is transmitted to the first unit cells of the bismuth ferrite film. Tilt angles in the $\mathrm{BiFeO}_{3}$ layer almost recover their bulk values at $\sim 10$ u.c. ahead of the interface. Similarly, the propagation of the octahedral tilt pattern of a rhombohedral $\mathrm{LaAlO}_{3}$ substrate into an epitaxial cubic $\mathrm{SrTiO}_{3}$ film has been directly determined using the negative spherical aberration imaging technique (Jia et al., 2009). These experiments provide direct evidence for the coupling of the antiferrodistortive order parameter (octahedral tilting) at interfaces, previously postulated for the $\mathrm{SrTiO}_{3} / \mathrm{LaTiO}_{3}$ (Hamann et al., 2006; Okamoto et al., 2006) and $\mathrm{SrTiO}_{3} / \mathrm{LaAlO}_{3}$ systems (Willmott et al., 2007) systems. Notably, a similar octahedral tilt suppression has also been determined inside the twin walls of $\mathrm{BiFeO}_{3}$ (Borisevich et al., 2010b), thus pointing an increased orbital overlap and thus bigger bandwidth as responsible for their enhanced conductivity (Catalan and Scott, 2009).

\section{SELF-ORGANIZATION OF NANOSTRUCTURES}

Island growth in heteroepitaxial systems is driven by high interface energy and high film surface energy. In octahedral framework systems, the flexibility of the lattice to accommodate large interfacial dissimilarities favors the wetting of the substrate hindering the formation of islands. However, interesting results have been obtained by the combination of different structural families using chemical solution deposition, like, for instance, perovskite/rock-salt $\left(\mathrm{La}_{0.7} \mathrm{Sr}_{0.3} \mathrm{MnO}_{3} / \mathrm{MgO}\right)$ (Abellán et al., 2012), fluorite/perovskite $\left(\mathrm{CeO}_{2} / \mathrm{LaAlO}_{3}\right)$ (Gibert et al., 2010), and perovskite/fluorite $\left(\mathrm{La}_{0.7} \mathrm{Sr}_{0.3} \mathrm{MnO}_{3} / \mathrm{YSZ}\right.$ ) (Zabaleta et al., 2013). In all cases, interfaces appear highly dislocated and therefore affected by strain fields that may extend over a few unit cells into the island with negative effects on their functionalities, such as the induction of polarization instabilities in ferroelectric materials (Chu et al., 2004). Interestingly, dislocation free $\mathrm{PbTiO}_{3}$ nanoislands exhibiting spontaneous polarization have been grown under a small tensile misfit strain of $0.15 \%$ on conducting $\mathrm{Nb}$-doped $\mathrm{SrTiO}_{3}$, using block copolymer micelles followed by epitaxial crystallization (Kim et al., 2010). The strong sensitivity of the growth mode to interfacial subtleties was demonstrated in the pulsed laser deposited Y-stabilized $\mathrm{ZrO}_{2} / \mathrm{SrTiO}_{3}$ (fluorite/perovskite) system, in which the fluorite film form continuous films or islands depending on the atomic termination plane of the $\mathrm{SrTiO}_{3}$ substrate (Cavallaro et al., 2011).

On the other hand, it has been recently reported that the extent of interfacial perturbations along the growth direction can be controlled through the growth kinetics, inducing morphological instabilities, which ultimately lead to self-organized surfaces. In particular, robust patterns of self-organized nanopits have been obtained on the surface of $\mathrm{La}_{0.7} \mathrm{Sr}_{0.3} \mathrm{MnO}_{3}$ films grown on $\mathrm{SrTiO}_{3}$ substrates (see Figure 2A) (Konstantinovic et al., 2013). The nanopits are aligned with the steps of the underlying substrate and result from the morphological evolution of continuous grooves created during the first growth stages to relax the strain concentration at step edges. In contrast with the mechanism operating in semiconductors, such as InGaN-based heterostructures, where nanopits form at the apex of threading dislocations (de Sousa Pereira et al., 2008) or associated to other strain sources embedded in the volume of the film (Liliental-Weber et al., 1997), here the formation of the nanopits does not require the presence of other defects, which could deteriorate the properties of the film. Moreover, the nanostructured surface has been successfully employed as a template for the integration of epitaxial $\mathrm{Au}$ nanocrystals exhibiting a perfect octahedral shape (Figures 2B-D) and excellent metallic-like behavior across the $\mathrm{Au} / \mathrm{La}_{0.7} \mathrm{Sr}_{0.3} \mathrm{MnO}_{3}$ interface (Figure 2E). Interestingly, such films exhibiting a modified distortion not found in conventional films, exhibit enhanced colossal magnetoresistance response (Pomar et al., 2014), thus further exemplifying the wide range of applications that can be accessed through the control of octahedral frameworks.

\section{FUTURE PROSPECTS}

Last decade has witnessed the emergence of unprecedented phenomena at transition-metal oxide interfaces that could not have been anticipated from the knowledge built upon conventional semiconductor epitaxy. This behavior stems from the confluence 

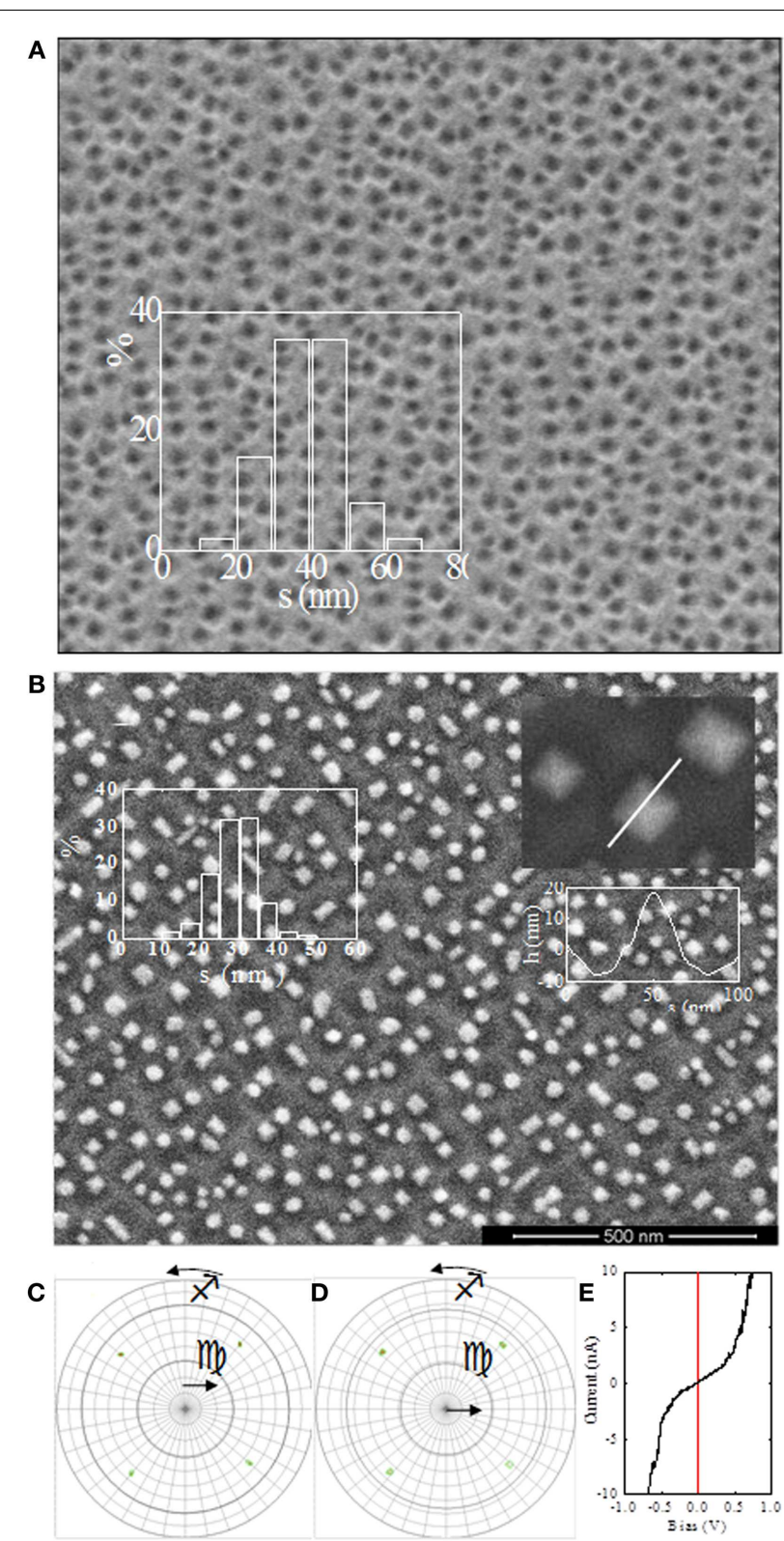

FIGURE 2 | (A) Scanning electron micrograph showing self-organized nanopits on the surface of $\mathrm{La}_{0.7} \mathrm{Sr}_{0.3} \mathrm{MnO}_{3}$ film. Inset shows the nanopit size distribution histogram. (B) Scanning electron micrograph of the Au nanocrystals grown inside the nanopits. Upper left inset shows the size distribution histogram of the Au nanocrystals. Upper right inset: upper panel is a magnified image of an Au nanocrystal exhibiting octahedral shape; lower panel shows a typical atomic force microscopy topographic profile of one Au nanocrystal. (C,D) (111)-X-ray diffraction pole-figures corresponding to the $\mathrm{La}_{0.7} \mathrm{Sr}_{0.3} \mathrm{MnO}_{3}$ film and the Au nanocrystals, demonstrating their cube-on-cube epitaxial relationship. (E) $I-V$ curves obtained by using atomic force microscopy in current sensing mode when the conducting tip is placed directly on top of the bare $\mathrm{La}_{0.7} \mathrm{Sr}_{0.3} \mathrm{MnO}_{3}$ surface (black curve) or on top of an Au nanocrystal (red curve). The black curve exhibits the typical features of tunneling conduction, resulting from the deterioration of the $\mathrm{La}_{0.7} \mathrm{Sr}_{0.3} \mathrm{MnO}_{3}$ surface, while the red curve exhibits metallic-like behavior, indicating that the Au nanocrystals protect the $\mathrm{La}_{0.7} \mathrm{Sr}_{0.3} \mathrm{MnO}_{3}$ surface against degradation due to ambient conditions (from Konstantinovic et al., 2013). of strongly competing charge, spin, orbital and lattice degrees of freedom, the coupling between them, and a surprisingly versatile mechanical response of octahedral frameworks to external perturbations. A radically new concept that holds strong promise for the predictive manipulation of the framework topology is the translation of the octahedral pattern of the substrate to the film (Rondinelli et al., 2012). Nevertheless, detailed knowledge on the boundaries between elastic, electronic, octahedral tilting, and plastic deformation regimes (Sandiumenge et al., 2013), as well as on the ability to control polar discontinuities (Dagotto, 2009; Boschker et al., 2012), is still needed to avoid interactions that could interfere with octahedral couplings. Besides electronic bandwidth-dependent properties, preferential orbital occupancies, particularly at free surfaces, open the door to catalytic applications (Suntivich et al., 2011a,b). An almost unexplored field is the manipulation of the octahedral framework to induce growth instabilities ultimately leading to self-organized surfaces (Konstantinovic et al., 2013), interfaces, or modulated bulk structures. On the other hand, guided organization using block copolymer micelles has been successfully used to limit the lateral dimensions of ferroelectric islands under almost null misfit strain (Kim et al., 2010). There is no doubt that octahedral frameworks still demand new challenging conceptual approaches toward nanofabrication and interface manipulation, but are becoming the working horse of many future applications.

\section{ACKNOWLEDGMENTS}

This research was supported by Spanish MEC (MAT201129081 and MAT2012-33207), CONSOLIDER (CSD2007-00041 and CSD2008-00023), and FEDER program.

\section{REFERENCES}

Abellán, P., Sandiumenge, F., Moreno, C., Casanove, M.-J., Puig, T., and Obradors, X. (2009). Strain relaxation of self-nanostructured solution derived $\mathrm{La}_{0.7} \mathrm{Sr}_{0.3} \mathrm{MnO}_{3}$ films. MRS Proc. 1174, doi:10.1557/PROC- 1174-V04- 12

Abellán, P., Zabaleta, J., Santiso, J., Casanove, M.-J., Dix, N., Aguiar, J., et al. (2012). Interface structure governed by plastic and structural dissimilarity in perovskite $\mathrm{La}_{0.7} \mathrm{Sr}_{0.3} \mathrm{MnO}_{3}$ nanodots on rock-salt $\mathrm{MgO}$ substrates. Appl. Phys. Lett. 100, 083104. doi:10.1063/1.3687692

Alderson, A., and Evans, K. (2002). Molecular origin of auxetic behavior in tetrahedral framework silicates. Phys. Rev. Lett. 89, 225503. doi:10.1103/PhysRevLett. 89.225503

Borisevich, A. Y., Chang, H. J., Huijben, M., Oxley, M. P., Okamoto, S., Niranjan, M. K., et al. (2010a). Suppression of octahedral tilts and associated changes in electronic properties at epitaxial oxide heterostructure interfaces. Phys. Rev. Lett. 105, 087204. doi:10.1103/PhysRevLett.105.087204

Borisevich, A. Y., Ovchinnikov, O. S., Chang, H. J., Oxley, M. P., Yu, P., Seidel, J., et al. (2010b). Mapping octahedral tilts and polarization across a domain wall in $\mathrm{BiFeO}_{3}$ from $\mathrm{Z}$-contrast scanning transmission electron microscopy image atomic column shape analysis. ACS Nano 4, 6071. doi:10.1021/nn1011539

Boschker, H., Verbeeck, J., Egoavil, R., Bals, S., van Tendeloo, G., Huijben, M., et al. (2012). Preventing the reconstruction of the polar discontinuity at oxide heterointerfaces. Adv. Funct. Mater. 22, 2235-2240. doi:10.1002/adfm.201102763

Calderon, M. J., Brey, L., and Guinea, F. (1999). Surface electronic structure and magnetic properties of doped manganites. Phys. Rev. B 60, 6698-6704. doi:10.1103/PhysRevB.60.6698

Catalan, G., and Scott, J. F. (2009). Physics and applications of bismuth ferrite. $A d v$. Mater. 21, 2463-2485. doi:10.1002/adma.200802849

Cavallaro, A., Ballesteros, B., Bachelet, R., and Santiso, J. (2011). Heteroepitaxial orientation control of YSZ films by selective growth on $\mathrm{SrO}-, \mathrm{TiO}_{2}-$ terminated $\mathrm{SrTiO}_{3}$ crystal surfaces. CrystEngComm. 13, 1625-1631. doi:10.1039/ C0CE00606H 
Chu, M.-W., Szafraniak, I., Scholz, R., Harnagea, C., Hesse, D., Alexe, M., et al. (2004). Impact of misfit dislocations on the polarization instability of epitaxial nanostructure ferroelectric perovskites. Nat. Mater. 3, 87-90. doi:10.1038/nmat1057

Dabrowski, B., Xiong, X., Bukowski, Z., Dybzinski, R., Klamut, P. W., Siewenie, J. E., et al. (1999). Structure-properties phase diagram for La1-xSrxMnO3 $(0.1 \leq \mathrm{x} \leq 0.2)$. Phys. Rev. B 60, 7006. doi:10.1103/PhysRevB.60.7006

Dagotto, E. (2009). Playing with the geometry of oxide heterostructures. Physics 2, 12-13. doi:10.1103/Physics.2.12

Daumont, C. J. M., Farokhipoor, S., Ferri, A., Wojdel, J. C., Íñiguez, J., Kooi, B. J., et al. (2010). Tuning the atomic and domain structure of epitaxial films of multiferroic BiFeO3. Phys. Rev. B 81, 144115. doi:10.1103/PhysRevB.81.144115

de Sousa Pereira, S. M., Martins, M. A., Trindade, T., Watson, I. M., Zhu, D., and Humphreys, C. J. (2008). Controlled integration of nanocrystals in inverted hexagonal nano-pits at the surface of light-emitting heterostructures. Adv. Mater. 20, 1038-1043. doi:10.1002/adma.200701739

Fang, Z., Solovyev, I., and Terakura, K. (2000). Phase diagram of tetragonal manganites. Phys. Rev. Lett. 84, 3169-3172. doi:10.1103/PhysRevLett.84.3169

Farag, N., Bobeth, M., Pompe, W., Romanov, A. E., and Speck, J. S. (2005). Modeling of twinning in epitaxial (001)-oriented $\mathrm{La}_{0.67} \mathrm{Sr}_{0.33} \mathrm{MnO}_{3}$ thin films. J. Appl. Phys. 97, 113516. doi:10.1063/1.1914950

Fitting, L., Thiel, S., Schmehl, A., Mannhart, J., and Muller, D. A. (2006). Subtleties in ADF imaging and spatially resolved EELS: a case study of low-angle grain boundaries in $\mathrm{SrTiO}_{3}$. Ultramicroscopy 106, 1053-1061. doi:10.1016/j.ultramic. 2006.04.019

Garcia, V., Bibes, M., Bocher, L., Valencia, S., Kronast, F., Crassous, A., et al. (2010). Ferroelectric control of spin polarization. Science 327, 1106-1110. doi:10.1126/science. 1184028

Gibert, M., Abellán, P., Benedetti, A., Puig, T., Sandiumenge, F., García, A., et al. (2010). Self-organized $\mathrm{Ce}_{1-\mathrm{x}} \mathrm{Gd}_{\mathrm{x}} \mathrm{O}_{2-\mathrm{y}}$ nanowire networks with very fast coarsening driven by attractive elastic interactions. Small 6, 2716-2724. doi:10.1002/ smll.201001237

Glazer, A. M. (1972). The classification of tilted octahedra in perovskites. Acta Cryst. B 28, 3384-3392. doi:10.1107/S0567740872007976

Gozar, A., Logvenov, G., Fitting Kourkoutis, L., Bollinger, A. T., Giannuzzi, L. A., Muller, D. A., et al. (2008). High-temperature interface superconductivity between metallic and insulating copper oxides. Nature 455, 782-785. doi:10.1038/nature07293

Hamann, D. R., Muller, D. A., and Hwang, H. Y. (2006). Lattice-polarization effects on electron-gas charge densities in ionic superlattices. Phys. Rev. B 73, 195403. doi:10.1103/PhysRevB.73.195403

Haun, M. J., Furman, E., Halemane, T. R., and Cross, L. E. (1989). Thermodynamic theory of the lead zirconate-titanate solid solution system, part IV: tilting of the oxygen octahedra. Ferroelectrics 99, 55-62. doi:10.1080/00150198908221436

Howard, J., and Stokes, H. T. (1998). Group-theoretical analysis of octahedral tilting in perovskites. Acta Cryst. B 54, 782-789. doi:10.1107/S0108768198004200

Huijben, M., Martin, L. W., Chu, Y. H., Holcomb, M. B., Yu, P., Rjinders, G., et al. (2008). Critical thickness and orbital ordering in ultrathin $\mathrm{La}_{0.7} \mathrm{Sr}_{0.3} \mathrm{MnO}_{3}$. Phys. Rev. B 78, 094413. doi:10.1103/PhysRevB.78.094413

Hwang, H. Y., Iwasa, Y., Kawasaki, M., Keimer, B., Nagaosa, N., and Tokura, Y. (2012). Emergent phenomena at oxide interfaces. Nat. Mater. 11, 103-113. doi:10.1038/nmat3223

Jia, C. L., Mi, S. B., Faley, M., Poppe, U., Schubert, J., and Urban, K. (2009). Oxygen octahedron reconstruction in the $\mathrm{SrTiO} 3 / \mathrm{LaAlO} 3$ heterointerfaces investigated using aberration-corrected ultrahigh-resolution transmission electron microscopy. Phys. Rev. B 79, 081405. doi:10.1103/PhysRevB.79.081405

Kim, Y., Han, H., Kim, Y., Lee, W., Alexe, M., Baik, S., et al. (2010). Untrahigh density array of epitaxial nanoislands on conducting substrates. Nano Lett. 10, 2141-2146. doi:10.1021/nl100819d

Konstantinovic, Z., Sandiumenge, F., Santiso, J., Balcells, L. L., and Martínez, B. (2013). Self-assembled pit arrays as templates for the integration of Au nanocrystals in oxide surfaces. Nanoscale 5, 1001-1008. doi:10.1039/c2nr33181k

Lee, J.-S., Arena, D. A., Yu, P., Nelson, C. S., Fan, R., Kinane, C. J., et al. (2010). Hidden magnetic configuration in epitaxial $\mathrm{La}_{1-\mathrm{x}} \mathrm{Sr}_{\mathrm{x}} \mathrm{MnO}_{3}$ films. Phys. Rev. Lett. 105, 257204. doi:10.1103/PhysRevLett.105.257204

Liliental-Weber, Z., Chen, Y., Ruvimov, S., and Washburn, J. (1997). Formation mechanism of nanotubes in GaN. Phys. Rev. Lett. 79, 2835-2838. doi:10.1103/ PhysRevLett.79.2835
Matthews, J. W. (1975). Defects associated with the accommodation of misfit between crystals. J. Vac. Sci. Technol. 12, 126-133. doi:10.1116/1.568741

Megaw, H. D., and Darlington, C. N. W. (1975). Geometrical and Structural relations in the rhombohedral perovskites. Acta Cryst. A 31, 161-173. doi:10.1107/ S0567739475000332

Moon, E. J., Balachandran, P. V., Kirby, B. J., Keavney, D. J., Sichel-Tissot, R. J., Schlepütz, C. M., et al. (2014). Effect of interfacial octahedral behavior in ultrathin manganite films. Nano Lett. 14, 2509-2514. doi:10.1021/nl500235f

Nakagawa, N., Hwang, H. Y., and Muller, D. A. (2006). Why some interfaces cannot be sharp. Nat. Mater. 5, 204-209. doi:10.1038/nmat1569

Ohtomo, A., and Hwang, H. Y. (2004). A high-mobility electron gas at the LaAlO3/SrTiO3 heterointerface. Nature 427, 423-426. doi:10.1038/nature02308

Okamoto, S., Millis, A. J., and Spaldin, N. (2006). Lattice relaxation in oxide heterostructures: $\mathrm{LaTiO}_{3} / \mathrm{SrTiO}_{3}$ superlattices. Phys. Rev. Lett. 97, 056802. doi:10.1103/PhysRevLett.97.056802

Pesquera, D., Herranz, G., Barla, A., Pellegrin, E., Bondino, F., Magnano, E., et al. (2012). Surface symmetry-breaking and strain effects on orbital occupancy in transition metal perovskite epitaxial films. Nat. Commun. 3:1189. doi:10.1038/ncomms2189

Pomar, A., Santiso, J., Sandiumenge, F., Roqueta, J., Bozzo, B., Frontera, C. et al. (2014). Growth kinetics engineered magnetoresistance response in $\mathrm{La}_{0.7} \mathrm{Sr}_{0.3} \mathrm{MnO}_{3}$ thin films. Appl. Phys. Lett. 104, 152406. doi:10.1063/1.4871984

Reyren, N., Thiel, S., Caviglia, A. D., Fitting Kourkoutis, L., Hammerl, G., Richter, C., et al. (2007). Superconducting interfaces between insulating oxides. Science 317, 1196-1199. doi:10.1126/science.1146006

Riedl, T. H., Gemming, T. H., Dörr, K., Luysberg, M., and Wetzig, K. (2009). $\mathrm{Mn}$ valency at $\mathrm{La} 0.7 \mathrm{Sr} 0.3 \mathrm{MnO} 3 / \mathrm{SrTiO} 3(001)$ thin film interfaces. Microsc. Microanal. 15, 213-221. doi:10.1017/S1431927609090229

Rondinelli, J. M., May, S. J., and Freeland, J. W. (2012). Control of octahedral connectivity in perovskite oxide heterostructures: an emerging route to multifunctional materials discovery. MRS Bull. 37, 261-270. doi:10.1557/mrs.2012.49

Rondinelli, J. M., and Spaldin, N. A. (2011). Structure and properties of functional oxide thin films: insights from electronic-structure calculations. Adv. Mater. 23, 3363-3381. doi:10.1002/adma.201101152

Sandiumenge, F., Puig, T., Rabier, J., Plain, J., and Obradors, X. (2000). Optimization of flux pinning in bulk melt textured 1-2-3 superconductors: bringing dislocations under control. Adv. Mat. 12, 375-381. doi:10.1002/(SICI)15214095(200003)12:5<375::AID-ADMA375>3.0.CO;2-9

Sandiumenge, F., Santiso, J., Balcells, L. L., Konstantinovic, Z., Roqueta, J., Pomar, A., et al. (2013). Competing misfit relaxation mechanisms in epitaxial correlated oxides. Phys. Rev. Lett. 110, 107206. doi:10.1103/PhysRevLett.110.107206

Santiso, J., Balcells, L. L., Konstantinovic, Z., Roqueta, J., Ferrer, P., Pomar, A., et al. (2013). Thickness evolution of the twin structure and shear strain in LSMO films. CrystEngComm. 15, 3908-3918. doi:10.1039/c3ce40085a

Suntivich, J., Gasteiger, H. A., Yabuuchi, N., Goodenough, J. B., and Shao-Horn, Y. (2011a). Design principles for oxygen-reduction activity on perovskite oxide catalysts for fuel cells and metal-air batteries. Nat. Chem. 3, 546-550. doi:10.1038/nchem.1069

Suntivich, J., May, K. J., Gasteiger, H. A., Goodenough, J. B., and Shao-Horn, Y. A. (2011b). Perovskite oxide optimized for oxygen evolution catalysis from molecular orbital principles. Science 334, 1383-1385. doi:10.1126/science.1212858

Szot, K., Speier, W., Bihlmayer, G., and Waser, R. (2006). Switching the electrical resistance of individual dislocations in single-crystalline $\mathrm{SrTiO}_{3}$. Nat. Mater. 5, 312-320. doi:10.1038/nmat1614

Tebano, A., Aruta, C., Sanna, S., Medaglia, P. G., Balestrino, G., Sidorenko, A. A., et al. (2008). Evidence of orbital reconstruction at interfaces in ultrahin $\mathrm{La}_{0.67} \mathrm{Sr}_{0.33} \mathrm{MnO}_{3}$ film. Phys. Rev. Lett. 100, 137401. doi:10.1103/PhysRevLett. 100.137401

Tokura, Y., and Nagaosa, N. (2000). Orbital physics in transition-metal oxides. Science 288, 462-468. doi:10.1126/science.288.5465.462

Urushibara, Y., Morimoto, Y., Arima, T., Asamitsu, A., Kido, G., and Tokura, Y. (1995). Insulator-metal transition and giant magnetoresistance in $\mathrm{La}_{1-\mathrm{x}} \mathrm{Sr}_{\mathrm{x}} \mathrm{MnO}_{3}$. Phys. Rev. B 51, 14103. doi:10.1103/PhysRevB.51.14103

Willmott, P. R., Pauli, S. A., Herger, R., Schlepütz, C. M., Martoccia, D., Patterson, B. D., et al. (2007). Structural basis for the conducting interface between $\mathrm{LaAlO}_{3}$ and $\mathrm{SrTiO}_{3}$. Phys. Rev. Lett. 99, 155502. doi:10.1103/ PhysRevLett.99.155502 
Zabaleta, J., Valencia, S., Kronast, F., Moreno, C., Abellán, P., Gázquez, J., et al. (2013). Photoemission electron microscopy study of sub-200 nm selfassembled $\mathrm{La}_{0.7} \mathrm{Sr}_{0.3} \mathrm{MnO}_{3}$ epitaxial islands. Nanoscale 5, 2990-2998. doi:10. 1039/c3nr33346a

Conflict of Interest Statement: The authors declare that the research was conducted in the absence of any commercial or financial relationships that could be construed as a potential conflict of interest.

Received: 27 June 2014; accepted: 07 August 2014; published online: 21 August 2014.
Citation: Sandiumenge F, Bagués $N$ and Santiso J (2014) Interfaces and nanostructures of functional oxide octahedral framework structures. Front. Mater. 1:13. doi: 10.3389/fmats.2014.00013

This article was submitted to Colloidal Materials and Interfaces, a section of the journal Frontiers in Materials.

Copyright (C) 2014 Sandiumenge, Bagués and Santiso. This is an open-access article distributed under the terms of the Creative Commons Attribution License (CC BY). The use, distribution or reproduction in other forums is permitted, provided the original author(s) or licensor are credited and that the original publication in this journal is cited, in accordance with accepted academic practice. No use, distribution or reproduction is permitted which does not comply with these terms. 